\title{
Propiedades mecánicas de un sistema epoxi modificado con ABS
}

\author{
L. BARRAL, J. CANO, J. LÓPEZ, P. NOGUEIRA, M.J. ABAD, A. TORRES Y C. RAMÍREZ \\ Departamento de Física, E.U.P. Ferrol. Universidad de A Coruña. Avda. 19 de Febrero s/n, 15405 Ferrol
}

\begin{abstract}
En este trabajo se ha estudiado la variación en las propiedades mecánicas de un sistema epoxi al modificarlo con diferentes contenidos de termoplástico (ABS). Los ensayos mecánicos fueron realizados a tres temperaturas distintas para investigar la influencia de esta variable en el comportamiento mecánico de los sistemas. Se observó que el aumento de temperatura del ensayo producía un incremento en la ductilidad del sistema. Las propiedades mecánicas del sistema no parecen experimentar gran mejoría con el incremento de la cantidad de termoplástico en el material. El alto grado de entrecruzamiento alcanzado durante el ciclo de curado puede limitar el efecto del ABS en el comportamiento mecánico del sistema epoxi.

Palabras clave: Epoxi, ABS, propiedades mecánicas, ductilidad.

Mechanical properties of an epoxy system modified with ABS

In this work, the effect of a thermoplastic modifier (ABS) on the mechanical properties of an epoxy resin (DGEBA) cured with a diamine (1,3-BAC) was studied. The experiments were carried out at three different temperatures. The effect of the temperature on the mechanical behaviour has been investigated too. It was observed that the rise in temperature improved the ductility of the modified epoxy systems. The presence of ABS did not produce a clear enhancement in the toughening of the epoxy resin, this behaviour was explained as a function of the high crosslink density reached in the curing cycle.
\end{abstract}

Key words: Epoxy, ABS, mechanical properties, ductility.

\section{INTRODUCCIÓN}

Las resinas epoxi tienen muchas aplicaciones industriales debido a que una vez curadas tienen gran rigidez, resistencia a la deformación, alta estabilidad térmica y buena resistencia química. También es sabido que estas resinas, altamente entrecruzadas, muestran un comportamiento quebradizo dependiendo de la estructura química de los componentes del sistema de la estequiometría utilizada y del proceso de curado (1).

Para intentar modificar este comportamiento, en las últimas décadas han surgido los materiales termoplásticos como modificadores alternativos a los elastómeros líquidos (CTBN y ATBN), en un intento de mejorar las propiedades mecánicas de los sistemas epoxi entrecruzados sin renunciar al excelente comportamiento térmico que los caracteriza.

Son muchos los termoplásticos que han sido utilizados como modificadores de los sistemas epoxi, entre ellos polietersulfonas (2), polisulfonas (3) y polieterimidas (4).

En este trabajo se utilizó un termoplástico mezcla de acrilonitrilo, butadieno y estireno (ABS) como modificador del sistema epoxi basado en una resina bifuncional curada con una diamina.

\section{PROCEDIMIENTO EXPERIMENTAL}

\subsection{Materiales}

La resina epoxi utilizada fue el diglicidil éter de bisfenol A (DGEBA), conocida comercialmente como Araldite GY 260 de Ciba. En la determinación del equivalente epoxi mediante análisis químico, se obtuvo un valor de $205.1 \mathrm{~g} / \mathrm{eq}$. Como endurece- dor fue empleado una diamina cicloalifática, el 1,3-bisaminometilciclohexano (1,3-BAC) de Aldrich Chemical, con una masa molecular de 142.25 y un grado de pureza del $99 \%$.

El termoplástico que fue añadido al sistema epoxi, ABS, se denomina comercialmente Polidux QI-300 de Polidux. Se utilizó la formulación estequiométrica resina/endurecedor, esto es, $100 \mathrm{~g}$ de resina epoxi por cada $17 \mathrm{~g}$ de diamina. El sistema epoxi fue modificado con 0, 5, 10 y 15 phr (partes por 100 de resina) de ABS.

\subsection{Preparación de las muestras}

Todos los componentes utilizados son productos comerciales sin purificación previa. Antes de empezar a mezclar, el ABS sufrió un proceso de desecación en el horno a $70^{\circ} \mathrm{C}$. El termoplástico fue disuelto en diclorometano, a esta mezcla se le añadió la resina DGEBA, a temperatura ambiente. El disolvente de la mezcla fue retirado colocando la mezcla en un baño térmico entre 80 y $100^{\circ} \mathrm{C}$, removiendo periódicamente con una varilla de vidrio. Por último se le añadió el endurecedor a temperatura ambiente y se homogeneizó el producto agitando manualmente.

La mezcla se vertió en un molde rectangular de vidrio previamente impregnado de una grasa desmoldeante y se sometió al ciclo de curado siguiente: 2 horas a temperatura ambiente, 5 horas a $40^{\circ} \mathrm{C}, 8$ horas a $60^{\circ} \mathrm{C}$ y un postcurado de 2 horas a $120^{\circ} \mathrm{C}$.

Las planchas obtenidas de dimensiones 14 x $30 \mathrm{~cm}^{2}$ y 4 $\mathrm{mm}$ de espesor fueron mecanizadas para obtener las probetas tipo M-II cuyas dimensiones vienen fijadas por la norma ASTM D638M (5). 


\subsection{Técnicas}

El análisis de las propiedades mecánicas del sistema epoxi modificado con ABS fue realizado mediante ensayos tensióndeformación llevados a cabo en una máquina universal de ensayos, Instron 5566, equipada con una cámara climática Instron 3119-005 refrigerada con $\mathrm{CO}_{2}$. La velocidad de deformación elegida fue de $1 \mathrm{~mm} / \mathrm{min}$. Se estudiaron las variaciones en las propiedades mecánicas del sistema epoxi a tres temperaturas $-20^{\circ} \mathrm{C}, 23^{\circ} \mathrm{C}$ y $75^{\circ} \mathrm{C}$. En todos los ensayos se siguió la norma ASTM D638M.

\section{RESULTADOS Y DISCUSIÓN}

\subsection{Efecto de la temperatura}

En un sistema epoxi se puede obtener desde el comportamiento típico de un material frágil y quebradizo hasta una curva tensión-deformación propia de elastómeros sólo con variar suficientemente la temperatura del ensayo (6).

De los ensayos de tracción realizados, bajo norma, a $-20^{\circ} \mathrm{C}$, $23^{\circ} \mathrm{C}$ y $75^{\circ} \mathrm{C}$, se obtuvieron los datos de la tensión y deformación en el punto de rotura, tensión y deformación en el punto de fluencia, el módulo elástico y la tenacidad de los tres sistemas modificados con ABS y del sistema neto.

La figura 1 muestra como varía la forma de la curva tensióndeformación al aumentar la temperatura de ensayo. La gráfica representa tres probetas del sistema modificado con $10 \mathrm{phr}$ de ABS, rotas a $-20^{\circ} \mathrm{C}, 23^{\circ} \mathrm{C}$ y $75^{\circ} \mathrm{C}$.

Para los cuatro sistemas epoxi estudiados (con 0, 5, 10 y 15 phr de ABS) el incremento en la temperatura de ensayo produce un aumento en la ductilidad del material, es decir, el módulo elástico disminuye, la deformación a la rotura aumenta y en los ensayos a $75^{\circ} \mathrm{C}$ aparecen puntos de fluencia en las curvas. La tenacidad del sistema, en general, se incrementa con el aumento de la temperatura (tabla I).

El aumento de la temperatura del ensayo no afectó de igual forma a los cuatro sistemas. El sistema modificado con $10 \mathrm{phr}$ de ABS mostró grandes disminuciones en el módulo de Young y en la tensión en el punto de rotura en los ensayos hechos a $75^{\circ} \mathrm{C}$. Este comportamiento puede explicarse debido a la proximidad de la temperatura de la transición vítrea del sistema $\left(\mathrm{T}_{\mathrm{g}}\right)$ a $75^{\circ} \mathrm{C}$. Como se puede observar en la tabla II, los tres sistemas modificados con ABS mostraron una única $\mathrm{T}_{\mathrm{g}}$ en el rango de temperatura estudiado. Las propiedades mecánicas del sistema son alteradas debido al aumento de la movilidad de las cadenas a temperaturas próximas a la $\mathrm{T}_{\mathrm{g}}$.

\subsection{Efecto del ABS en las propiedades mecánicas del sistema}

El añadir termoplástico al sistema epoxi no alteró su naturaleza "frágil" y "quebradiza", con pequeñas deformaciones porcentuales y tensiones altas en el punto de rotura. Las gráficas de la figura 2 muestran como varía la tensión y la deformación en el punto de rotura, a una temperatura dada, con el contenido de ABS. El módulo elástico o de Young obtenido en los ensayos a una temperatura dada, permanece prácticamente invariante al aumentar el porcentaje de termoplástico en las probetas (ver figura 3). La única excepción es la que presentan las probetas con $10 \mathrm{phr}$ de $\mathrm{ABS}$ en los ensayos a $75^{\circ} \mathrm{C}$, con una brusca disminución en el valor del módulo debido a la proximidad de la $\mathrm{T}_{\mathrm{g}}$. En los experimentos realizados a temperatura subambiente $\left(\mathrm{T}=-20^{\circ} \mathrm{C}\right)$ se observó un aumento de la deformación y la tensión máxima en el punto de rotura para las
TABLA I. VARIACIÓN CON LA TEMPERATURA DE LA TENACIDAD DE LOS SISTEMAS EPOXI MODIFICADOS CON ABS.

\begin{tabular}{|l|l|l|l|l|}
\hline \multicolumn{5}{|c|}{ Tenacidad (MPa) } \\
\hline Epoxi/ABS & $\mathbf{0 ~} \mathbf{~ h r}$ & $\mathbf{5} \mathbf{~ p h r}$ & $\mathbf{1 0} \mathbf{~ p h r}$ & $\mathbf{1 5} \mathbf{~ h r}$ \\
\hline $\mathbf{- 2 0 ^ { \circ } \mathbf { C }}$ & 0.2 & 0.4 & 0.6 & 0.2 \\
\hline $\mathbf{2 3 ^ { \circ } \mathbf { C }}$ & 1.1 & 0.8 & 0.5 & 0.4 \\
\hline $\mathbf{7 5}^{\circ} \mathbf{C}$ & 2.0 & 0.9 & 0.8 & 1.4 \\
\hline
\end{tabular}

TABLA II. TEMPERATURA DE TRANSICIÓN VÍtREA DE LOS SISTEMAS, MEDIDAS CON DSC A VELOCIDAD DE CALENTAMIENTO CONSTANTE DE $10^{\circ} \mathrm{C} / \mathrm{MIN}$.

\begin{tabular}{|l|l|}
\hline Epoxi/ABS & $\mathbf{T}_{\mathbf{g}}\left({ }^{\circ} \mathbf{C}\right)$ \\
\hline $0 \mathrm{phr}$ & 115.5 \\
\hline $5 \mathrm{phr}$ & 104.7 \\
\hline $10 \mathrm{phr}$ & 69.4 \\
\hline $15 \mathrm{phr}$ & 87.9 \\
\hline
\end{tabular}

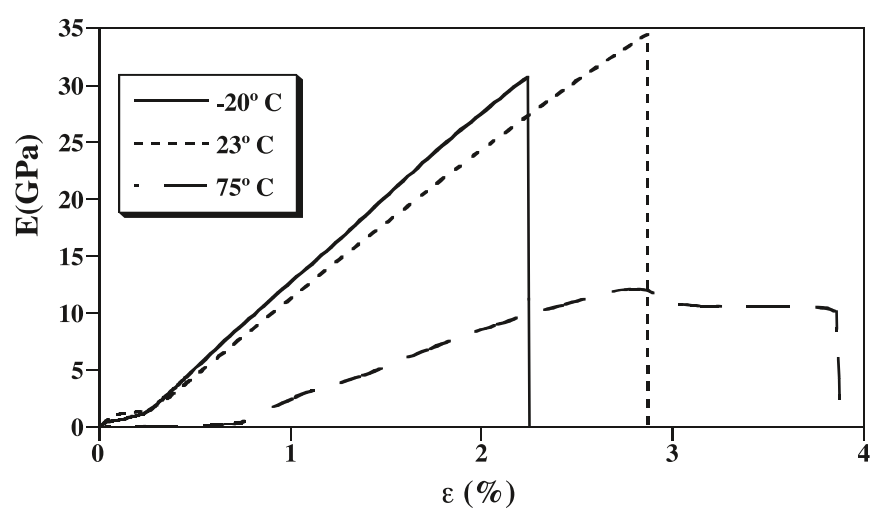

Figura 1. Curvas tensión-deformación para el sistema epoxi con 10 phr de ABS.

probetas con 5 y 10 phr de ABS, respecto al sistema neto. La modificación con 15 phr de termoplástico no resultó efectiva a esa temperatura de ensayo y los valores de las propiedades obtenidos son similares a los del sistema neto.

Para los experimentos realizados a temperatura ambiente, si consideramos el margen de error estadístico, no aparecen variaciones en las propiedades mecánicas con la cantidad de ABS presente en el sistema.

A $75^{\circ} \mathrm{C}$ todos los sistemas mostraron puntos de fluencia en las curvas tensión-deformación, señal de que el material es más dúctil a esa temperatura.

El margen de error estadístico que aparece en todos los datos obtenidos en los ensayos no permiten definir si hay una mejoría o un empeoramiento de las propiedades. Sólo en el caso de las muestras con 10 phr de ABS se ve una clara disminución de los valores de la tensión, tanto en el punto de rotura como en el de fluencia, debido a la proximidad de la $\mathrm{T}_{\mathrm{g}}$ a la temperatura de $75^{\circ} \mathrm{C}$.

De los resultados obtenidos se puede entrever que la modificación del sistema epoxi no ha resultado claramente efectiva, en parte porque el ciclo de curado al que fue sometido el material no resulta el más idóneo. La red está demasiado entrecruzada y por lo tanto muy rígida como para permitir mejorías en el comportamiento mecánico. Posiblemente un curado menos severo permitiría observar cambios importantes en las propiedades mecánicas del sistema al modificarlo con ABS. 


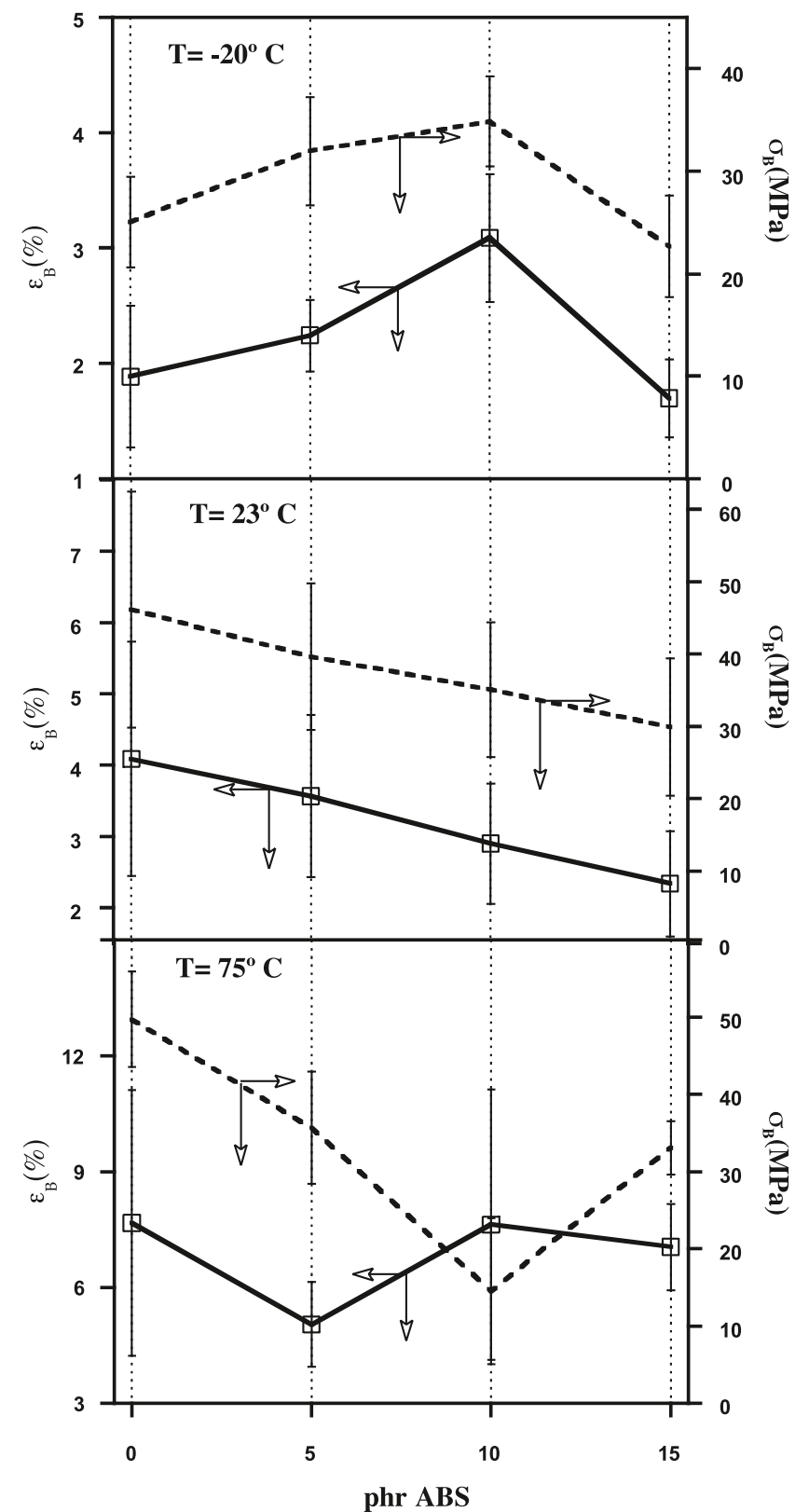

Figura 2. Variación de $\varepsilon_{\mathrm{B}}$ y $\sigma_{\mathrm{B}}$ con el contenido de $\mathrm{ABS}$ a distintas temperaturas de ensayo.

\section{CONCLUSIONES}

El principal objetivo de este trabajo es el estudio de la variación de las propiedades mecánicas de un sistema epoxi DGEBA/1,3-BAC modificado con distintas cantidades de ABS. Los ensayos mecánicos fueron realizados a tres temperaturas diferentes para estudiar también la influencia de esta variable en el comportamiento mecánico.

Las conclusiones obtenidas se pueden resumir en lo siguiente:

El comportamiento mecánico del sistema se ve más alterado por la temperatura de ensayo que por el aumento de termoplástico en la mezcla.

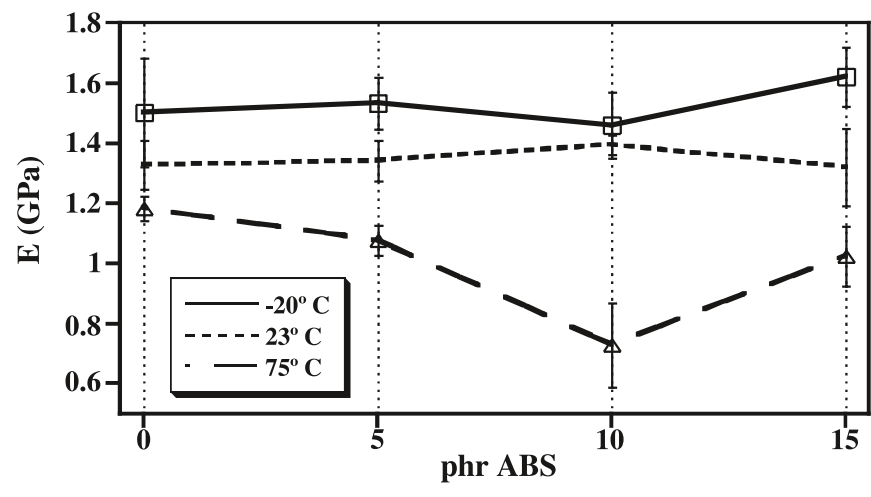

Figura 3. Variación del módulo elástico o de Young con el contenido de ABS y la temperatura de ensayo.

El incremento de la temperatura conduce a una comportamiento más dúctil en los materiales, es decir, aumenta la deformación en el punto de rotura, se incrementa la tenacidad y los módulos decrecen ligeramente. Las muestras con 10 phr de ABS presentan sorprendentes disminuciones en las propiedades mecánicas medidas a $75^{\circ} \mathrm{C}$ debido a la proximidad de la $\mathrm{T}_{\mathrm{g}}$ del sistema a esa temperatura.

Con el aumento de la cantidad de ABS en el sistema epoxi no se observaron sustanciales mejorías en las propiedades mecánicas. Por una parte las altas desviaciones estadísticas, que inevitablemente aparecen en los ensayos, no permiten distinguir un comportamiento claro en la variación de los datos y por otro lado, el curado al que se sometió el material no parece el más idóneo, ya que la red está excesivamente entrecruzada.

\section{AGRADECIMIENTOS}

Este trabajo ha sido financiado con cargo al proyecto subvencionado por la Consellería de Educación de la Xunta de Galicia XUGA-17201B97.

\section{BIBLIOGRAFÍA}

1. L. Barral, J. Cano, J. López, P. Nogueira y C. Ramírez. “Effect of Thermal Degradation on the Mechanical Properties of a Diglycidyl Ether of Bisphenol A/1,3-Bisaminomethylcyclohexane (DGEBA/1,3-BAC) Epoxy Resin System", J. Appl. Polym. Sci. 63, 1841-1849 (1997).

2. C.B. Bucknall y I.K. Partridge. "Phase Separation in Epoxy Resins Containing Polyethersulphone". Polymer, 24, 639-644 (1983).

3. E.M. Woo, L.D. Bravenec y J.C. Seferis. "Morphology and Properties of an Epoxy Alloy System Containing Thermoplastics and a Reactive Rubber", Polym. Eng. Sci. 34, 1664-1673 (1994).

4. E. Girard-Reydet, V. Vicard, J.P. Pascault y H. Sautereau. "PolyetherimideModified Epoxy Networks: Influence of Cure Conditions on Morphology and Mechanical Properties", J. Appl. Polym. Sci. 65, 2433-2445 (1997).

5. ASTM D638M, 1994 Annual Book of ASTM Standards, ASTM Section 8, Plastics, Ed. Staff, USA (1994).

6. L.E. Nielsen y R.F. Landel en Mechanical Properties of Polymers and Composites, Ed. Marcel Dekker, 1994. 